\title{
Complexe de poids des variétés algébriques réelles avec action
}

\author{
Fabien Priziac
}

\begin{abstract}
Résumé
En utilisant la fonctorialité du complexe de poids de C. McCrory et A. Parusiński qui induit un analogue de la filtration par le poids pour les variétés algébriques complexes sur l'homologie de Borel-Moore à coefficients dans $\mathbb{Z}_{2}$ des variétés algébriques réelles-, on définit un complexe de poids avec action sur les variétés algébriques réelles munies d'une action d'un groupe fini. Mettant l'accent sur le groupe à deux éléments, on établit ensuite une version filtrée de la suite courte de Smith pour une involution, tenant compte de la filtration Nash-constructible qui réalise le complexe de poids avec action. Son exactitude est impliquée par le découpage d'une variété Nash munie d'une involution algébrique le long d'un sous-ensemble symétrique par arcs.
\end{abstract}

\section{Introduction}

Dans [3], P. Deligne a établi l'existence d'une filtration dite par le poids sur la cohomologie rationnelle des variétés algébriques complexes. Un analogue de cette filtration sur l'homologie de Borel-Moore à coefficients dans $\mathbb{Z}_{2}$ des variétés algébriques réelles a été introduit par $\mathrm{B}$. Totaro dans [17. En utilisant un critère d'extension de foncteurs définis sur les variétés lisses de F. Guillén et V. Navarro Aznar ([6]), C. McCrory et A. Parusiński ont montré dans [14 l'existence d'un complexe de chaînes filtré nommé de poids, défini à quasi-isomorphisme filtré près, fonctoriel sur la catégorie des variétés algébriques réelles et induisant la filtration par le poids de Totaro. La suite spectrale de poids réelle ne converge pas dès l'ordre deux (contrairement à son homologue complexe), et McCrory et Parusiński mettent en évidence la richesse des informations qu'elle contient -par exemple les nombres de Betti virtuels ([13])-, tout en en enrichissant la compréhension, en réalisant par exemple le complexe de poids dès le niveau des chaînes par une filtration géométrique. Leur filtration Nash-constructible -définie en utilisant les fonctions Nash-constructibles- l'étend à la catégorie des ensembles et morphismes $\mathcal{A S}$ ([9], [10]), établissant la fonctorialité des objets "de poids" par rapport aux applications continues avec graphe $\mathcal{A S}$.

Mots-clés : filtration par le poids, variétés algébriques réelles, action de groupe, suite exacte de Smith, ensembles symétriques par arcs, fonctions Nash-constructibles, variétés Nash. 2010 Mathematics Subject Classification : 14P25, 14P10, 14P20, 57S17, 57S25

Travail issu d'une thèse effectuée au laboratoire IRMAR (Institut de Recherche Mathématique de Rennes), au sein de l'Université de Rennes 1, et partiellement financé par le projet ANR-08-JCJC-0118-01. 
Dans cet article, on considère des variétés algébriques réelles munies de l'action d'un groupe fini. On met alors à profit la fonctorialité du complexe de poids pour le munir de l'action induite (théorème 3.7). D'une part, la finitude du groupe, impliquant l'existence d'une résolution des singularités équivariante, d'une compactification équivariante et d'un lemme de ChowHironaka équivariant (4], remarque 3.6), nous permet de montrer, en utilisant une version avec action du critère d'extension de Guillén et Navarro Aznar (théorème 3.5), l'unicité (à quasi-isomorphisme filtré équivariant près) du complexe de poids avec action. D'autre part, l'équivariance des opérations de base sur les chaînes semi-algébriques à supports fermés nous permet de le réaliser via la filtration géométrique/Nash-constructible, munie de l'action induite également par fonctorialité (remarque 3.9).

Dans la suite de l'article, on souhaite comprendre plus en avant les chaînes invariantes sous l'action, et notamment la compatibilité de la suite exacte courte de Smith avec la filtration Nash-constructible. Pour cela, on se concentre sur le cas du groupe à deux éléments, pour lequel cette étude se révèle déjà très intéressante. On montre ainsi que l'on peut avoir un certain contrôle sur la régularité du découpage d'une chaîne invariante proposé par la suite exacte de Smith d'une involution. Précisément, on établit que, pour tout entier $\alpha$, on peut écrire une chaîne invariante de degré $\alpha$ (vis-à-vis de la filtration Nash-constructible) comme la somme de sa restriction à l'ensemble des points fixes, d'une chaîne de degré au plus $\alpha+1$ et de l'image de celle-ci par l'involution (proposition 5.2).

Pour parvenir à ce résultat, on réduit la question au cas d'une variété Nash affine compacte connexe munie d'une involution algébrique (non triviale). On prouve qu'un tel objet peut être découpé le long d'un sous-ensemble symétrique par arcs en deux morceaux semi-algébriques échangés sous l'action (théorème 4.1).

Enfin, en guise d'interprétation de cette suite exacte courte de Smith Nash-constructible, on montre que dans le cas d'une variété algébrique compacte munie d'une action libre, l'isomorphisme entre les chaînes invariantes et les chaînes du quotient, qui est alors symétrique par arcs, est compatible avec la filtration Nash-constructible (proposition 5.6).

On débute cet article en munissant, dans la partie2, le complexe des chaînes semi-algébriques à supports fermés d'une variété algébrique réelle munie d'une action de groupe, de l'action induite. On remarque alors l'équivariance de la fonctorialité des chaînes semi-algébriques, ainsi que celle des opérations de restriction, d'adhérence et de tiré en arrière.

C'est dans la partie 3 que le complexe de poids des variétés algébriques réelles avec action d'un groupe fini est muni de l'action induite par fonctorialité. Y est également établie son unicité à quasi-isomorphisme filtré équivariant près, en utilisant l'analogue avec action du critère d'extension de Guillén et Navarro Aznar.

L'existence d'un découpage d'une variété Nash munie d'une involution algébrique le long d'un sous-ensemble symétrique par arcs est énoncée et démontrée dans la partie 4. On l'utilise ensuite dans la partie 5 pour établir notre suite exacte courte de Smith Nash-constructible, pour laquelle on y donne aussi notre interpétation dans le cas compact sans point fixe.

Remerciements. L'auteur souhaite remercier M. Coste, G. Fichou, F. Guillén, T. Limoges, C. McCrory et A. Parusiński pour de fructueux discussions et commentaires. 


\section{Chaînes semi-algébriques et action de groupe}

Considérant une action sur un ensemble semi-algébrique localement compact, on munit le complexe de ses chaînes semi-algébriques à supports fermés (14] Appendix) de l'action induite par fonctoralité. On vérifie alors que l'action commute avec les opérations de restriction, d'adhérence (proposition 2.4) et de tiré en arrière (proposition 2.5).

Dans cet article, suivant la définition de [14, une variété algébrique réelle désignera un schéma réduit séparé de type fini sur $\mathbb{R}$.

Soit donc $X$ un sous-ensemble semi-algébrique de l'ensemble des points réels d'une variété algébrique réelle. Pour la commodité du lecteur, on rappelle la définition du complexe des chaînes semi-algébriques à supports fermés de $X$ ([14] Appendix) :

Définition 2.1. Pour tout $k \geq 0$, on note $C_{k}(X)$ le quotient $d u \mathbb{Z}_{2}$-espace vectoriel engendré par les sous-ensembles semi-algébriques fermés de $X$ de dimension $\leq k$, par les relations

- la somme $A+B$ est équivalente à l'adhérence dans $X$ (pour la topologie forte) $c l_{X}(A \div B$ ) de la différence symétrique de $A$ et de $B$,

- la classe de A est nulle si la dimension de A est strictement plus petite que $k$.

Une chaîne semi-algébrique à supports fermés de dimension $k$ de $X$ est une classe d'équivalence de $C_{k}(X)$. Toute chaîne $c$ de $C_{k}(X)$ peut s'écrire comme la classe d'un sous-ensemble semialgébrique fermé $A$ de $X$ de dimension $\leq k$, notée $[A]$.

L'opérateur de bord $\partial_{k}: C_{k}(X) \rightarrow C_{k-1}(X)$ est défini par, si c $=[A] \in C_{k}(X), \partial_{k} c=[\partial A]$ où $\partial A$ désigne le bord semi-algébrique $\left\{x \in A \mid \Lambda \mathbf{1}_{A}(X) \equiv 1 \bmod 2\right\}$ de $A$ ( $\Lambda$ est l'entrelacs sur les fonctions constructibles : cf [12]).

On renvoie à l'appendice de [14] pour les définitions du poussé en avant, des opérations de restriction et d'adhérence, ainsi que celle du tiré en arrière.

On suppose maintenant que $X$ est muni d'une action d'un groupe $G$ qui agit par homéomorphismes semi-algébriques : pour tout $g \in G$, on note $\alpha_{g}: X \rightarrow X$ l'homéomorphisme semi-algébrique associé à $g$.

Par fonctorialité, on obtient alors une action sur le complexe $C_{*}(X)$ par isomorphismes linéaires donnée par $\alpha_{g_{*}}: C_{*}(X) \rightarrow C_{*}(X)$ pour $g \in G$.

Le complexe $C_{*}(X)$ muni de cette action de $G$ devient alors un $G$-complexe.

Lemme 2.2. Soient $Y$ un autre ensemble semi-algébrique localement compact muni d'une action de $G$ et $f: X \rightarrow Y$ une application semi-algébrique continue propre et équivariante. Alors le poussé en avant $f_{*}$ est équivariant par rapport aux actions de $G$ sur les $G$-complexes $C_{*}(X)$ et $C_{*}(Y)$.

Démonstration. Fonctorialité des chaînes semi-algébriques à supports fermés ([14] Appendix).

L'action de $G$ sur une chaîne semi-algébrique à support fermé peut être donnée par l'action sur l'un de ses représentants : 
Proposition 2.3. Soient $g \in G$ et $c=[A] \in C_{k}(X)$, alors

$$
g . c=[g \cdot A] .
$$

Démonstration. On a $g . c:=\alpha_{g_{*}}(c)=[B]$, où

$$
B=\operatorname{cl}\left\{x \in X \mid \alpha_{g_{*}} \mathbf{1}_{A}(x)=\chi\left(\alpha_{g}^{-1}(x) \cap A\right) \equiv 1 \bmod 2\right\} .
$$

Or pour $x \in X, \chi\left(\alpha_{g}^{-1}(x) \cap A\right)=1$ si $\alpha_{g}^{-1}(x) \in A$ i.e. $x \in \alpha_{g}(A), 0$ si $\alpha_{g}^{-1}(x) \notin A$ i.e. $x \notin \alpha_{g}(A)$ (car $\alpha_{g}$ est un homéomorphisme). Donc $B=c l\left(\alpha_{g}(A)\right)=\alpha_{g}(A)$.

A partir de cette constatation, on peut montrer simplement la commutativité de l'action avec les opérations de restriction, d'adhérence et le tiré en arrière :

Proposition 2.4. Soit $Z \subset X$ un sous-ensemble semi-algébrique localement fermé globalement stable sous l'action de $G$. La restriction à $Z$ des chaînes semi-algébriques à supports fermés de $X$ commute avec l'action induite de G. De même, l'opération d'adhérence des chaînes semialgébriques à support fermés de $Z$ commute avec l'action induite de $G$.

Démonstration. Soient $k \geq 0$ et $c=[A] \in C_{k}(X)$. Alors,

$$
g \cdot\left(\left.c\right|_{Z}\right)=\left[\alpha_{g}(A \cap Z)\right]=\left[\alpha_{g}(A) \cap Z\right]=\left.(g . c)\right|_{Z} .
$$

Soient $k \geq 0$ et $c=[A] \in C_{k}(Z)$. Alors,

$$
\overline{g . c}=\left[\operatorname{cl}\left(\alpha_{g}(A)\right]=\left[\alpha_{g}(c l(A))\right]=g \cdot \bar{c} .\right.
$$

Proposition 2.5. On considère le diagramme commutatif d'ensembles semi-algébriques localement fermés suivant :

$$
\begin{array}{lll}
\tilde{Y} & \rightarrow & \tilde{X} \\
\downarrow & & \downarrow \pi \\
Y & \stackrel{i}{\rightarrow} & X
\end{array}
$$

où $\pi: \widetilde{X} \rightarrow X$ est une application semi-algébrique continue propre, $i$ est l'inclusion d'un sousensemble semi-algébrique fermé, $\tilde{Y}=\pi^{-1}(Y)$, et la restriction $\pi: \widetilde{X} \backslash \widetilde{Y} \rightarrow X \backslash Y$ est un homéomorphisme, et où tous les objets sont munis d'une action de $G$ et tous les morphismes sont équivariants par rapport à ces actions.

L'action de $G$ sur les chaînes semi-algébriques à supports fermés commute alors avec le tiré en arrière.

Démonstration. L'action de $G$ commute avec l'adhérence, le poussé en avant, la restriction donc avec le tiré en arrière.

On établit enfin deux autres lemmes, utilisés dans la preuve du corollaire 5.2. Dans ce résultat fondamental de notre étude, on parviendra à découper une chaîne semi-algébrique invariante sous une involution, modulo sa restriction aux points fixes, en deux chaînes images l'une de l'autre et dont le "degré de régularité" (par rapport à la filtration dite Nash-constructible de [14]) ne s'éloignera pas trop du degré initial de la chaîne de départ. 
Lemme 2.6. Soient $A_{1}, A_{2}$ et $A_{3}$ trois sous-ensembles semi-algébriques fermés de $X$ de dimension k. Si $\left[A_{1}\right]=\left[A_{2}\right]$ dans $C_{k}(X)$, alors $\left[A_{1} \cap A_{3}\right]=\left[A_{2} \cap A_{3}\right]$.

Démonstration. On a $\left[A_{1} \cap A_{3}\right]+\left[A_{2} \cap A_{3}\right]=\left[\operatorname{cl}\left(\left(A_{1} \cap A_{3}\right) \div\left(A_{2} \cap A_{3}\right)\right)\right]$, or $\left(A_{1} \cap A_{3}\right) \div$ $\left(A_{2} \cap A_{3}\right)=\left(A_{1} \div A_{2}\right) \cap A_{3}$ et $\operatorname{dim}\left(A_{1} \div A_{2}\right)<k$ car $\left[A_{1}\right]=\left[A_{2}\right]$.

Lemme 2.7. Soient $A_{1}, A_{2}$ deux sous-ensembles semi-algébriques de $X$ de dimension $k$ avec $A_{1}$ fermé dans $X$. Alors, dans $C_{k}(X),\left[\operatorname{cl}\left(A_{1} \cap A_{2}\right)\right]=\left[A_{1} \cap \operatorname{cl}\left(A_{2}\right)\right]$.

Démonstration. On a

$\operatorname{cl}\left(A_{1} \cap A_{2}\right) \div\left(A_{1} \cap \operatorname{cl}\left(A_{2}\right)\right)=A_{1} \cap \operatorname{cl}\left(A_{2}\right) \backslash \operatorname{cl}\left(A_{1} \cap A_{2}\right) \subset A_{1} \cap \operatorname{cl}\left(A_{2}\right) \backslash A_{1} \cap A_{2}=A_{1} \cap\left(\operatorname{cl}\left(A_{2}\right) \backslash A_{2}\right)$

et $\operatorname{dim}\left(c l\left(A_{2}\right) \backslash A_{2}\right)<k$.

\section{Complexe de poids avec action}

Soit $G$ un groupe fini.

On définit (théorème 3.7) un complexe de poids avec action de $G$ sur la catégorie de ce que l'on appellera les $G$-variétés algébriques réelles, fonctoriel par rapport aux morphismes propres réguliers équivariants, et unique à quasi-isomorphisme filtré équivariant près avec les propriétés d'extension, d'acyclicité et d'additivité analogues à celles du cadre sans action de [14] Theorem 1.1. Pour son existence, cela consistera à munir le complexe de poids de McCrory-Parusiński de l'action de $G$ induite par fonctorialité. Quant à son unicité, elle sera donnée par une version avec action du critère d'extension de F. Guillén et V. Navarro Aznar ([ $[$ ] Théorème 2.2.2), qui justifie la restriction au cas d'un groupe fini pour lequel il existe une compactification équivariante, un lemme de Chow-Hironaka équivariant ainsi qu'une résolution des singularités équivariante.

On prêtera ensuite attention au groupe $G=\mathbb{Z} / 2 \mathbb{Z}$. En effet, dès le plus petit groupe non trivial, les structures avec action s'enrichissent considérablement. Dans la section 5, on établit une version de la suite exacte courte de Smith qui tient compte de la filtration Nash-constructible (qui réalise le complexe de poids par [14] Theorem 2.8 et Corollary 3.11). Ce résultat repose sur un théorème de découpage des variétés Nash munies d'une involution algébrique, que l'on établit dans la section 4 .

Tout d'abord, définissons précisément les catégories sur lesquelles nous allons travailler. Dans toute la suite, une action de $G$ par isomorphismes algébriques sur une variété algébrique réelle $X$ désignera une action par isomorphismes de schémas telle que l'orbite de tout point de $X$ est contenue dans un sous-schéma ouvert affine.

Définition 3.1. On note

- $\mathbf{S c h}_{c}^{G}(\mathbb{R})$ la catégorie des variétés algébriques réelles munies d'une action de $G$ par isomorphismes algébriques -on nomme de tels objets des $G$-variétés algébriques réelles-et des morphismes propres réguliers équivariants,

- $\operatorname{Reg}_{\text {comp }}^{G}(\mathbb{R})$ la sous-catégorie des G-variétés compactes non singulières, 
- $\mathbf{V}^{G}(\mathbb{R})$ la sous-catégorie des $G$-variétés projectives non singulières.

On note également

- $\mathcal{C}^{G}$ la catégorie des $G$-complexes de $\mathbb{Z}_{2}$-espaces vectoriels bornés munis d'une filtration croissante bornée par des $G$-complexes avec inclusions équivariantes -on nomme de tels objets des $G$-complexes filtrés- et des morphismes de complexes filtrés équivariants,

- $\mathcal{D}^{G}$ la catégorie des $G$-complexes bornés et des morphismes de complexes équivariants.

Remarque 3.2. - Si $X$ est une $G$-variété algébrique réelle, on a vu que l'action de $G$ sur $X$ induit, par fonctorialité de $C_{*}: \mathbf{S c h}_{c}(\mathbb{R}) \rightarrow \mathcal{D} ; X \mapsto C_{*}(X):=C_{*}(X(\mathbb{R}))$, une action de $G$ $\operatorname{sur} C_{*}(X)$, et donc également une action (linéaire) sur l'homologie $H_{*}(X):=H_{*}\left(C_{*}(X)\right.$ ), qui est l'homologie de Borel-Moore de l'ensemble des points réels de $X$ à coefficients dans $\mathbb{Z}_{2}$ ([14] section 1$)$. On a ainsi un foncteur

$$
C_{*}: \operatorname{Sch}_{c}^{G}(\mathbb{R}) \rightarrow \mathcal{D}^{G} ; X \mapsto C_{*}(X) .
$$

- Si $\left(K_{*}, F_{*}\right)$ est un $G$-complexe filtré, la suite spectrale induite est naturellement munie d'une action de groupe : chaque terme est munie de l'action de $G$ induite, et les différentielles sont équivariantes pour celle-ci.

- Le complexe simple filtré associé à un diagramme cubique dans $\mathcal{C}^{G}$ ([14 section 1.1) peut être naturellement muni de l'action du groupe $G$ induite (en considérant l'action diagonale sur les sommes directes), et devenir ainsi un élément de $\mathcal{C}^{G}$.

Dans la continuité de ce qu'ont fait C. McCrory et A. Parusiński dans le cadre sans action de groupe, on s'intéresse aux morphismes filtrés qui induisent des isomorphismes au niveau des suites spectrales :

Définition 3.3. On note $\mathrm{Ho}_{\mathcal{C}^{G}}$ la catégorie $\mathcal{C}^{G}$ localisée par rapport aux quasi-isomorphismes filtrés équivariants, autrement appelés quasi-isomorphismes de $\mathcal{C}^{G}$, i.e. les morphismes filtrés équivariants entre $G$-complexes filtrés qui induisent un isomorphisme (équivariant) au niveau $E^{1}$ des suites spectrales induites.

Remarque 3.4. Tout $G$-complexe $K_{*}$ de $\mathcal{D}^{G}$ peut être muni de la filtration canonique ([14 section 1.1) sur laquelle agit naturellement le groupe $G$ (le noyau de la différentielle de $K_{*}$, qui est équivariante, est stable sous l'action de $G)$, et ainsi $\left(K_{*}, F_{*}^{c a n}\right)$ est un élément de $\mathcal{C}^{G}$.

De cette façon, un quasi-isomorphisme équivariant entre $G$-complexes bornés induit un quasi-isomorphisme filtré équivariant entre $G$-complexes filtrés munis de la filtration canonique.

Nous allons à présent pouvoir énoncer le théorème d'existence et d'unicité (dans $\mathrm{Ho}_{\mathrm{C}}{ }^{G}$ ) du complexe de poids avec action du groupe $G$.

L'existence sera donnée directement par la fonctorialité du complexe de poids (sans action) de C. McCrory et A. Parusiński. Son unicité requerra une version avec action du critère d'extension de F. Guillén et V. Navarro Aznar énoncé dans [6] :

Théorème 3.5. Soient $\mathcal{C}$ une catégorie de descente cohomologique et

$$
F: \mathbf{V}^{G}(\mathbb{R}) \longrightarrow \mathrm{Ho}_{\mathrm{C}}
$$

un foncteur contravariant $\Phi$-rectifié qui vérifie 
(F1) $F(\emptyset)=0$, et le morphisme canonique $F(X \sqcup Y) \rightarrow F(X) \times F(Y)$ est un isomorphisme (dans $\left.H_{0} \mathcal{C}\right)$,

(F2) si $X_{\bullet}$ est un carré acyclique élémentaire de $\mathbf{V}^{G}(\mathbb{R}), \mathbf{s} F\left(X_{\bullet}\right)$ est acyclique. Alors, il existe une extension de $F$ en un foncteur contravariant $\Phi$-rectifié

$$
F_{c}: \operatorname{Sch}_{c}^{G}(\mathbb{R}) \rightarrow H o \mathcal{C}
$$

telle que :

1. si $X_{\bullet}$ est un carré acyclique de $\mathbf{S c h}_{c}^{G}(\mathbb{R}), \mathbf{s} F_{c}\left(X_{\bullet}\right)$ est acyclique,

2. si $Y$ est une sous-variété fermée de $X$ stable sous l'action de $G$ sur $X$, on a un isomorphisme naturel (dans Ho $\mathcal{C}$ )

$$
F_{c}(X \backslash Y) \cong \mathbf{s}\left(F_{c}(X) \rightarrow F_{c}(Y)\right) .
$$

En outre, cette extension est unique, à isomorphisme unique près.

Remarque 3.6. Le critère d'extension ([6] Théorème 2.2.2) reste bien valable dans ce contexte. En effet, celui-ci nécessite, sur la catégorie de variétés considérée, une résolution des singularités, un lemme de Chow-Hironaka et une compactification. Or, sur la catégorie $\mathbf{S c h}_{c}^{G}(\mathbb{R})$, comme le groupe $G$ est d'ordre fini, une résolution des singularités équivariante, un lemme de ChowHironaka équivariant ainsi qu'une compactification équivariante existent par [4] (Appendix).

Théorème 3.7. Le foncteur

$$
F^{c a n} C_{*}: \mathbf{V}^{G}(\mathbb{R}) \longrightarrow H_{o} \mathcal{C}^{G} ; X \mapsto F^{c a n} C_{*}(X)
$$

admet une extension en un foncteur

$$
{ }^{G} \mathcal{W} C_{*}: \operatorname{Sch}_{c}^{G}(\mathbb{R}) \longrightarrow H o \mathcal{C}^{G}
$$

défini pour toutes les G-variétés algébriques réelles et tous les morphismes propres réguliers équivariants, qui vérifie les propriétés suivantes :

1. Acyclicité : Pour tout carré acyclique

$$
\begin{array}{lll}
\tilde{Y} & \rightarrow & \tilde{X} \\
\downarrow & & \downarrow \\
Y & \rightarrow & X
\end{array}
$$

dans $\mathbf{S c h}_{c}^{G}(\mathbb{R})$, le complexe filtré simple du $\square_{1}^{+}$-diagramme dans $\mathcal{C}^{G}$

$$
\begin{array}{ccc}
G_{\mathcal{W} C_{*}(\tilde{Y})} & \rightarrow & { }^{G} \mathcal{W} C_{*}(\tilde{X}) \\
\downarrow & & \downarrow \\
G_{\mathcal{W} C_{*}(Y)} & \rightarrow & G_{\mathcal{W}} C_{*}(X)
\end{array}
$$

est acyclique (i.e. isomorphe au complexe nul dans $\mathrm{Ho}_{\mathrm{C}}{ }^{G}$ ). 
2. Additivité : Pour une inclusion fermée équivariante $Y \subset X$, le complexe filtré simple du $\square_{0}^{+}$-diagramme dans $\mathcal{C}^{G}$

$$
{ }^{G} \mathcal{W} C_{*}(Y) \rightarrow{ }^{G} \mathcal{W} C_{*}(X)
$$

est isomorphe $\grave{a}^{G} \mathcal{W} C_{*}(X \backslash Y)$.

Un tel foncteur ${ }^{G} \mathcal{W} C_{*}$ est unique à un isomorphisme de $H_{o} \mathcal{C}^{G}$ unique près.

Démonstration. Existence. Soit $X$ une $G$-variété algébrique réelle. On a une action de $G$ sur le complexe filtré de poids $\mathcal{W} C_{*}(X)$ de $X$ induite par fonctorialité du complexe de poids.

De plus, tout morphisme propre régulier équivariant induira, toujours par fonctorialité de $\mathcal{W} C_{*}$, un morphisme de $H o \mathcal{C}$ équivariant, i.e. un morphisme de $H o \mathcal{C}^{G}$.

On obtient donc ainsi un foncteur ${ }^{G} \mathcal{W} C_{*}: \mathbf{S c h}_{c}^{G}(\mathbb{R}) \rightarrow H_{o} \mathcal{C}^{G}$, celui qui à toute $G$-variété algébrique réelle $X$ associe son complexe filtré de poids $\mathcal{W} C_{*}(X)$ muni de l'action induite.

Ce foncteur est bien une extension de $F^{c a n} C_{*}: \mathbf{V}^{G}(\mathbb{R}) \longrightarrow H o \mathcal{C}^{G}$ car le complexe de poids $\mathcal{W} C_{*}: \mathbf{S c h}_{c}(\mathbb{R}) \longrightarrow H_{o} \mathcal{C}$ est une extension de $F^{c a n} C_{*}: \mathbf{V}(\mathbb{R}) \longrightarrow H_{o} \mathcal{C}\left(\operatorname{pour} X \in \mathbf{V}^{G}(\mathbb{R})\right.$, l'action sur le complexe filtré $F^{c a n} C_{*}(X)$ est également induite par fonctorialité).

Il vérifie enfin les conditions d'acyclicité et d'additivité (le morphisme nul est équivariant et, dans le théorème 2.2.2 de [6] la condition d'additivité repose sur une égalité permettant l'extension du foncteur aux variétés non compactes, ce qui la rend a fortiori équivariante).

Unicité. Pour l'unicité, nous utiliserons le critère d'extension de Guillén-Navarro Aznar ([6] Théorème 2.2.2) adapté au cadre avec action du groupe $G$ que l'on a énoncé précédemment (théorème 3.5). Appliquons-le (tout du moins sa version homologique et covariante) à notre contexte pour obtenir l'unicité du complexe de poids avec action de $G$ :

$-\mathcal{C}^{G}$ est une catégorie de descente homologique, en tant que catégorie des complexes de chaînes de $\mathbb{Z}_{2}[G]$-modules à gauche (i.e. les $\mathbb{Z}_{2}$-espaces vectoriels munis d'une action linéaire du groupe $G$ ), bornés et munis d'une filtration croissante bornée. En effet, les $\mathbb{Z}_{2}[G]$-modules à gauche forment une catégorie abélienne et on utilise alors la propriété (1.7.5) de [6].

- Le foncteur $F^{c a n} C_{*}$ est $\Phi$-rectifié, car il est défini sur la catégorie $\mathcal{C}^{G}$.

- Le foncteur $F^{c a n} C_{*}$ vérifie les conditions (F1) et (F2) dans le cadre sans action. Munissant, dans la preuve de 14 Theorem 1.1, les variétés projectives lisses d'une action agébrique de $G$, tous les morphismes mentionnés, induits par l'application du foncteur $F^{c a n} C_{*}$, sont alors équivariants, et celui-ci vérifie donc bien les conditions (F1) et (F2) dans le cadre avec action de $G$.

Définition 3.8. Si $X$ est une $G$-variété algébrique réelle, le $G$-complexe filtré ${ }^{G} \mathcal{W} C_{*}(X)$ (défini à quasi-isomorphisme filtré équivariant près) est appelé le complexe de poids avec action de $X$.

Remarque 3.9. - L'isomorphisme $H_{n}\left({ }^{G} \mathcal{W} C_{*}(X)\right) \cong H_{n}(X)$ pour toute $G$-variété réelle $X$ (14] Proposition 1.5) est équivariant. En effet, dans la preuve de 14] Proposition 1.5, les foncteurs $C_{*}: \mathbf{S c h}_{c}(\mathbb{R}) \rightarrow H o \mathcal{D}$ et $\varphi \circ \mathcal{W} C_{*}: \mathbf{S c h}_{c}(\mathbb{R}) \rightarrow H o \mathcal{D}$ sont isomorphes. 
Ainsi, si $X$ est une $G$-variété algébrique réelle, le quasi-isomorphisme entre $\varphi \circ \mathcal{W} C_{*}(X)$ et $C_{*}(X)$ est équivariant par rapport aux actions induites par fonctorialité.

- La filtration par le poids et la suite spectrale de poids se retrouvent munies de l'action induite du groupe $G$.

- La filtration géométrique/Nash-constructible de McCrory et Parusiński ([14] sections 2 et 3 ), munie de l'action de $G$ induite par fonctorialité, réalise le complexe de poids avec action des $G$-variétés algébriques réelles. En effet, lorsque l'on munit les variétés considérées d'une action algébrique de $G$, les morphismes des suites exactes courtes de 14 Theorem 2.7 et Theorem 3.6 sont équivariants pour les actions induites, ainsi que les inclusions $\mathcal{G}_{p} C_{k}(X) \subset F_{p}^{c a n} C_{k}(X)$ pour toute $G$-variété réelle $X$.

- Comme dans le cadre sans action (14 Proposition 1.8), le complexe de poids ${ }^{G} \mathcal{W} C_{*}(X)$ avec action de $G$ d'une variété compacte non singulière est quasi-isomorphe dans $\mathcal{C}^{G}$ à $F^{c a n} C_{*}(X)$. Il en va de même pour toutes ses réalisations.

Dans la suite, le complexe de poids avec action d'une $G$-variété réelle $X$ sera simplement noté $\mathcal{W} C_{*}(X)$ lorsque le contexte sera explicite.

\section{Le découpage d'une variété Nash affine compacte munie d'une action de $G=\mathbb{Z} / 2 \mathbb{Z}$}

On souhaite découper toute chaîne invariante d'une variété algébrique réelle avec action de $G=\mathbb{Z} / 2 \mathbb{Z}=\{1, \sigma\}$, après en avoir retiré la partie invariante point par point, en deux morceaux qui soient l'image l'un de l'autre par l'involution $\sigma$, mais des morceaux dont on puisse contrôler la régularité vis-à-vis de la filtration Nash-constructible.

On s'intéresse au cas particulier du groupe à deux éléments car il est le premier cas difficile, tant pour cette question particulière que pour l'étude des variétés algébriques réelles avec action de groupe en général.

L'ingrédient-clé qui nous permettra d'effectuer ce découpage sera le résultat suivant. Ce théorème affirme que l'on peut découper toute variété Nash affine compacte connexe munie d'une involution algébrique le long d'un sous-ensemble symétrique par arcs ([9], [10]), globalement invariant sous l'action. Une sous-variété Nash d'un espace affine $\mathbb{R}^{N}$ est une sous-variété $\mathcal{C}^{\infty}$ de $\mathbb{R}^{N}$ qui en est également un sous-ensemble semi-algébrique. On renvoie à [16] pour tout le matériel concernant les variétés Nash et les fonctions Nash, i.e les fonctions $\mathcal{C}^{\infty}$ semialgébriques.

Théorème 4.1. Soit $M$ une sous-variété Nash compacte connexe d'un espace affine $\mathbb{R}^{N}$ munie d'une involution algébrique $\sigma$ (i.e. la restriction d'une involution algébrique sur l'adhérence de Zariski de $M)$ non triviale. Alors il existe un sous-ensemble symétrique par arcs $S$ de $M$ de codimension 1 globalement stable sous l'action de $\sigma$, et un sous-ensemble semi-algébrique fermé $A$ de $M$ tels que $M=A \cup \sigma(A), S=A \cap \sigma(A)$ et $S=\partial A$. 


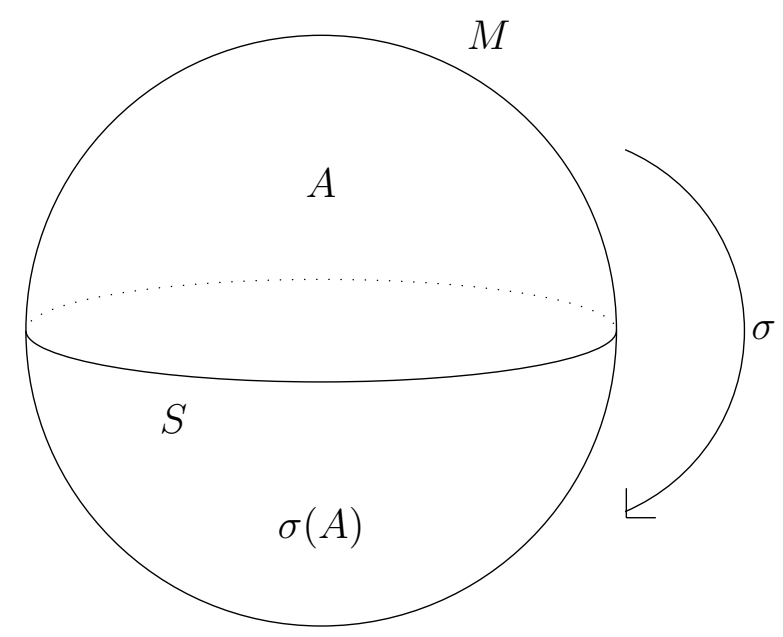

Démonstration. La démonstration de ce résultat va se baser sur l'obtention d'une application classifiante entre le quotient de $M$ privé de ses points fixes, que l'on notera $N$, par l'action de $G$ et un espace projectif réel. On approchera cette fonction, tout du moins son extension à la compactificaction Nash de $N / G$, via le théorème de Stone-Weierstrass et l'utilisation d'un voisinage tubulaire Nash ([1] Corollary 8.9.5), par une fonction Nash, transverse au sous-espace projectif de codimension 1, que l'on relèvera ensuite en une fonction équivariante définie sur $M \backslash M^{G}$ et à valeurs dans une sphère munie de l'action antipodale, Nash et transverse à la sphère de codimension 1 . Considérant alors l'image réciproque de celle-ci, ainsi que celles des deux hémisphères, auxquels on rajoute les points fixes, on parvient à découper $M$ en deux sousensembles semi-algébriques, images l'une de l'autre par l'involution, le long d'un sous-ensemble symétrique par arcs.

(i) Quotient. Considérons donc $N$ la variété $M$ privée de ses points fixes. $N$ est une sousvariété Nash de $\mathbb{R}^{N}$, de même dimension que $M$ (car l'action de $G$ sur $M$ n'est pas triviale) et munie d'une action libre de $G$ (où $G=\{i d, \sigma\}=\mathbb{Z} / 2 \mathbb{Z}$ ). Le quotient $N / G$ est une variété Nash. En effet, le quotient d'un ensemble semi-algébrique par l'action (semi-algébrique) d'un groupe est un ensemble semi-algébrique ([15]), et le quotient d'une variété (différentiable) lisse (i.e. $\left.\mathcal{C}^{\infty}\right)$ par l'action libre (et lisse) d'un groupe fini est une variété lisse ([1] Theorem 7.10). La variété Nash $N / G$ est de plus affine, en tant que sous-ensemble (semi-algébrique) de l'ensemble des points réels du quotient de la complexification de l'adhérence de Zariski de $M$, qui est une variété complexe projective, par la complexification de l'action de $G$, quotient qui est lui aussi une variété projective complexe.

(ii) Compactification. La variété $N / G$ étant une variété Nash affine, on peut la compactifier en une variété Nash affine avec bord d'après le théorème VI.2.1. de [16]. Plus précisément, il existe une variété algébrique affine compacte non-singulière $X^{\prime}$, une sous-variété algébrique 
non-singulière $Y^{\prime}$ de codimension 1 (vide si $N / G$ est compacte i.e. si $M^{G}=\emptyset$ ), une composante connexe $N^{\prime}$ de $X \backslash Y$ telles qu'il existe un difféomorphisme Nash $\psi: N / G \rightarrow N^{\prime}$ et $M^{\prime}:=\operatorname{cl}\left(N^{\prime}\right)$ est une variété Nash à bord compacte (connexe), de bord $Y$.

(iii) Application classifiante. Le groupe $G=\mathbb{Z} / 2 \mathbb{Z}$ agissant librement sur le variété lisse $N$, on peut considèrer le $\mathbb{Z} / 2 \mathbb{Z}$-fibré principal lisse $\Pi: N \rightarrow N / G$ ainsi qu'une application classifiante lisse $f: N / G \rightarrow \mathbb{P}^{M}(\mathbb{R})([8])$. Remarquons que "l" " application classifiante est définie à homotopie près. Aussi, notre but va être dès à présent de construire une application classifiante $h: N / G \rightarrow \mathbb{P}^{M}(\mathbb{R})$ qui sera Nash et transverse à $\mathbb{P}^{M-1}(\mathbb{R})$.

(iv) Extension. On commence pour cela par composer $f$ par $\psi^{-1}$ : on obtient une application lisse $f^{\prime}:=f \circ \psi^{-1}: N^{\prime} \rightarrow \mathbb{P}^{M}(\mathbb{R})$. Puis on étend à homotopie près l'application $f^{\prime}$ à la compactification Nash $M^{\prime}$ de $N^{\prime}$, ceci grâce à une propriété énoncée par M. Shiota dans [16]. L'application $f^{\prime}$ étant en effet une application continue entre $N^{\prime}=M^{\prime} \backslash \partial M^{\prime}$ et $\mathbb{P}^{M}(\mathbb{R})$, où $M^{\prime}$ et $\mathbb{P}^{M}(\mathbb{R})$ sont en particulier des variétés PL (piecewise-linear) compactes, le lemme V.1.4 de [16] nous dit que $f^{\prime}$ est homotope à la restriction à $N^{\prime}$ d'une application PL, et donc en particulier continue semi-algébrique, $\overline{f^{\prime}}: M^{\prime} \rightarrow \mathbb{P}^{M}(\mathbb{R})$. On va par la suite chercher à approcher la fonction $\overline{f^{\prime}}$ grâce à un voisinage tubulaire Nash.

(v) Approximations. Plongeons donc $M^{\prime}$ dans un espace affine $\mathbb{R}^{N_{0}}$ et $\mathbb{P}^{M}(\mathbb{R})$ dans un espace affine $\mathbb{R}^{M_{0}}$. On considère un voisinage tubulaire Nash $\left(U, \rho: U \rightarrow \mathbb{P}^{M}(\mathbb{R})\right)$ de $\mathbb{P}^{M}(\mathbb{R})$ dans $\mathbb{R}^{M_{0}}$ ([1] Corollary 8.9.5).

$M^{\prime}$ étant compact, on approche alors $h_{0}:=\overline{f^{\prime}}$ par une application polynomiale $h_{1}$ en utilisant le théorème de Stone-Weirstrass ([1] Theorem 8.8.5). Puis on approche l'application $h_{1}$ par une application lisse $h_{2}$ transverse à $\mathbb{P}^{M-1}(\mathbb{R})$. Enfin, cette dernière condition étant ouverte, on approche $h_{2}$ par une application polynomiale (en utilisant encore une fois StoneWeierstrass) de façon suffisamment proche pour obtenir une application $h_{3}$ qui soit polynomiale et transverse à $\mathbb{P}^{M-1}(\mathbb{R})$. Précisons que l'on effectue ces approximations de manière à ce qu'à chaque fois, pour tout $t \in[0,1]$ et pour tout $x \in M / G,(1-t) h_{i}(x)+t h_{i+1}(x) \in U$.

Considérant alors les homotopies successives

$$
(t, x) \mapsto \rho\left((1-t) h_{i}(x)+t h_{i+1}(x)\right),
$$

on obtient ainsi une homotopie entre $\overline{f^{\prime}}: M / G \rightarrow \mathbb{P}^{M}(\mathbb{R})$ et $\overline{h^{\prime}}:=\rho \circ h_{3}: M / G \rightarrow \mathbb{P}^{M}(\mathbb{R})$. L'application $\overline{h^{\prime}}$ est Nash et transverse à $\mathbb{P}^{M-1}(\mathbb{R})$, car $\rho$ est une submersion Nash (en tant que composition d'un difféomorphisme Nash et de la projection d'un fibré vectoriel Nash).

(vi) Restriction. Enfin, en restreignant l'homotopie à $N^{\prime}$ et en notant $h^{\prime}$ la restriction de $\overline{h^{\prime}}$ à $N^{\prime}$, on obtient une application

$$
h^{\prime}: N^{\prime} \rightarrow \mathbb{P}^{M}(\mathbb{R})
$$

Nash, transverse à $\mathbb{P}^{M-1}(\mathbb{R})$, homotope à $f^{\prime}$. En la composant enfin avec $\psi$ pour revenir au quotient $N / G$, on peut supposer que l'application

$$
h:=h^{\prime} \circ \psi: N / G \rightarrow \mathbb{P}^{M}(\mathbb{R})
$$


Nash, transverse à $\mathbb{P}^{M-1}(\mathbb{R})$ et homotope à $f=f^{\prime} \circ \psi$, est l'application classifiante associée au $\mathbb{Z} / 2 \mathbb{Z}$-fibré principal lisse $\Pi: N \rightarrow N / G$.

(vii) Relèvement. On relève alors $h$ en une application

$$
\widetilde{h}: N \rightarrow \mathbb{S}^{M}(\mathbb{R})
$$

équivariante (par rapport à l'action de $G$ sur $N$ et l'action antipodale sur $\mathbb{S}^{M}(\mathbb{R})$ ) transverse à $\mathbb{S}^{M-1}(\mathbb{R})$, analytique et localement semi-algébrique, donc Nash, car une application analytique localement semi-algébrique entre deux variétés Nash, dont celle d'arrivée est affine, est Nash (remarque $(\mathrm{xv})$ à la page 16 de [16]).

(viii) Découpage de $N$. On note

$-N^{+}:=\widetilde{h}^{-1}\left(S^{+}\right)$,

$-N^{-}:=\widetilde{h}^{-1}\left(S^{-}\right)=\sigma\left(N^{+}\right)$,

- $W:=\widetilde{h}^{-1}\left(\mathbb{S}^{M-1}(\mathbb{R})\right)=N^{+} \cap N^{-}$.

$\left(S^{+}\right.$et $S^{-}$sont respectivement les hémisphères nord et sud de la sphère $\mathbb{S}^{M}(\mathbb{R})$, échangés sous l'action antipodale).

$N^{+}$et $N^{-}$sont des sous-ensembles semi-algébriques fermés de $N$ de même dimension que $N$, et, par transversalité de l'application Nash $\widetilde{h}$ (et parce que $\mathbb{S}^{M-1}(\mathbb{R})$ est une sous-variété Nash de $\left.\mathbb{S}^{M}(\mathbb{R})\right), W$ est une sous-variété Nash de $N$ de codimension 1. C'est de plus un sous-ensemble $\mathcal{A S}$ de $N$, en tant qu'image réciproque du symétrique par $\operatorname{arcs} \mathbb{S}^{M-1}(\mathbb{R})$ par l'application analytique semi-algébrique $\widetilde{h}$.

On a bien évidemment $N^{+} \cup N^{-}=N$ mais on a également $\partial N^{+}=\partial N^{-}=W$.

En effet, le bord semi-algébrique d'un ensemble semi-algébrique $A$ est tout d'abord défini par

$$
\partial A=\{x \in A \mid \chi(A \cap S(x, \epsilon)) \equiv 1 \bmod 2\}
$$

pour $\epsilon$ suffisamment petit, or, la caractéristique d'Euler à supports compacts étant additive et $N$ et $W$ ne possédant pas de bord, on a modulo 2 , pour tout $x$ dans $N$,

$$
\begin{aligned}
0 & \equiv \chi(N \cap S(x, \epsilon)) \\
& \equiv \chi\left(N^{+} \cap S(x, \epsilon)\right)+\chi\left(N^{-} \cap S(x, \epsilon)\right)-\chi(W \cap S(x, \epsilon)) \\
& \equiv \chi\left(N^{+} \cap S(x, \epsilon)\right)+\chi\left(N^{-} \cap S(x, \epsilon)\right)
\end{aligned}
$$

Un élément $x$ de $N$ est ainsi dans le bord de $N^{+}$si et seulement s'il est dans le bord de $N^{-}$. En particulier, $\partial N^{+}=\partial N^{-} \subset N^{+} \cap N^{-}=W$.

Réciproquement, soit $x \in W$ et supposons par l'absurde que $x \notin \partial N^{+}=\partial N^{-}$. Le point $x$ appartient alors à $N^{+} \backslash \partial N^{+}$et $N^{-} \backslash \partial N^{-}$, qui sont des ouverts semi-algébriques de $N$ : il existe donc des ouverts semi-algébriques $U$ et $V$ de $N$ (de même dimension $n$ que $N$ ) tels que $x \in U \subset N^{+} \backslash \partial N^{+}$, et $x \in V \subset N^{-} \backslash \partial N^{-}$. L'ouvert $U \cap V$ de $N$, de dimension $n$ (non vide car contenant $x$ ), est alors inclus dans $\left(N^{+} \backslash \partial N^{+}\right) \cap\left(N^{-} \backslash \partial N^{-}\right)$qui est de dimension au plus $n-1$, en tant que sous-ensemble de $W$. 
(ix) Découpage de $M$. Enfin, on rajoute les points fixes en considérant l'adhérence de $N$ dans $M$, qui est $M$ tout entier car $M$ est une variété Nash connexe dont la sous-variété Nash $M^{G}$ est de codimension au moins 1 . On note alors

$-M^{+}:=\operatorname{cl}\left(N^{+}\right)$,

$-M^{-}:=\operatorname{cl}\left(N^{-}\right)=\sigma\left(M^{+}\right)$.

On voit immédiatement que $M^{+} \cup M^{-}=M$. Montrons que l'on a $M^{+} \cap M^{-}=W \cup M^{G}$. On prouve pour cela que $\operatorname{cl}\left(N^{+}\right) \backslash N^{+}=\operatorname{cl}\left(N^{-}\right) \backslash N^{-}=W \cup M^{G}$.

En effet, comme $\operatorname{cl}\left(N^{+}\right) \backslash N^{+} \subset M^{G}$,

$$
\operatorname{cl}\left(N^{+}\right) \backslash N^{+}=\sigma\left(\operatorname{cl}\left(N^{+}\right) \backslash N^{+}\right)=\operatorname{cl}\left(N^{-}\right) \backslash N^{-} .
$$

De plus,

$M=M^{+} \cup M^{-}=\left(N^{+} \cup N^{-}\right) \sqcup\left(\left(\operatorname{cl}\left(N^{+}\right) \backslash N^{+}\right) \cup\left(\operatorname{cl}\left(N^{-}\right) \backslash N^{-}\right)\right)=\left(M \backslash M^{G}\right) \sqcup\left(\operatorname{cl}\left(N^{+}\right) \backslash N^{+}\right)$

donc $\operatorname{cl}\left(N^{+}\right) \backslash N^{+}=M^{G}$. En particulier, $M^{+} \cap M^{-}=W \cup M^{G}$. Remarquons que, comme $W$ est un sous-ensemble $\mathcal{A S}$ de $M \backslash M^{G}, W \cup M^{G}$ est un sous-ensemble $\mathcal{A S}$ de $M$, en tant qu'union de deux tels ensembles (la catégorie $\mathcal{A S}$ est l'algèbre booléenne engendré par les sous-ensembles symétriques par arcs de $\mathbb{P}^{N}(\mathbb{R})$ ). Il est de plus fermé dans le compact $M$ et est donc symétrique par arcs.

Enfin, on montre que $\partial M^{+}=\partial M^{-}=W \cup M^{G}$, d'une manière identique à ce que l'on avait fait dans le cas de $N$.

Dans la section suivante 5, on va, comme annoncé, utiliser ce résultat pour pouvoir découper toute chaîne d'une variété algébrique réelle en deux morceaux suffisamment réguliers.

\section{La suite exacte courte de Smith Nash-constructible dans le cas $G=\mathbb{Z} / 2 \mathbb{Z}$}

Soit $X$ une variété algébrique réelle munie d'une involution algébrique $\sigma$. Dans cette partie, on montre que l'on peut écrire toute chaîne $c$ globalement invariante de $X$, de dimension $k$ et de degré $\alpha$ dans la filtration Nash-constructible (avec action), comme la somme

$$
c=c_{\mid X^{G}}+(1+\sigma) \gamma
$$

où $c_{\mid X^{G}} \in \mathcal{N}_{\alpha} C_{k}\left(X^{G}\right)$ est la restriction de $c$ à l'ensemble des points invariants de $X$ et où $\gamma \in \mathcal{N}_{\alpha+1} C_{k}(X)$.

Considérant une chaîne $c$, notre démarche va consister à découper en de tels morceaux l'adhérence de Zariski du support de $c$, puis à considérer les intersections du support de $c$ avec ceux-ci, qui répondront alors aux conditions recherchées. 
On procède ainsi en deux étapes. On traite tout d'abord le cas d'une chaîne pure, et ensuite celui d'une chaîne quelconque.

Pour la première étape, on se ramènera au cas où le support de la chaîne est une variété Nash compacte et on utilisera le théorème 4.1 de la section précédente, afin de découper une chaîne pure de $X$, invariante sous l'action de $G$, en deux chaînes dont le bord est pur :

Proposition 5.1. Soit $c \in\left(\mathcal{N}_{-k} C_{k}(X)\right)^{G}$ une chaîne pure de dimension $k$, invariante sous l'action de $G$. Alors il existe une chaîne $\gamma \in \mathcal{N}_{-k+1} C_{k}(X)$ telle que

$$
c=c_{\mid X^{G}}+(1+\sigma) \gamma
$$

(la restriction $c_{\mid X^{G}} \grave{a} X^{G}$ appartient $\grave{a} \mathcal{N}_{-k} C_{k}\left(X^{G}\right)$ ).

Démonstration. Notons tout d'abord que, $c$ étant une chaîne invariante sous l'action de $G$, le support de $c$ est également globalement invariant. En effet, si $A$ est un sous-ensemble semialgébrique de $X$ représentant la chaîne $c$, c'est également le cas pour $\sigma(A)$ et, comme l'involution $\sigma$ est un isomorphisme algébrique, on a

$$
\text { Supp } c=\left\{y \in \sigma(A) \mid \operatorname{dim}_{y} \sigma(A)=k\right\}=\sigma\left(\left\{x \in A \mid \operatorname{dim}_{x} A=k\right\}\right)=\sigma(\operatorname{Supp} c) .
$$

On va alors se ramener au cas où le support de $c$, que l'on note $M$, est une sous-variété Nash compacte connexe d'un espace affine.

(1) On peut supposer que la $G$-variété algébrique réelle $X$ est compacte : si ce n'est pas le cas, on considère une compactification équivariante $X_{0}$ de $X$ (4 Appendix), et l'adhérence $\bar{c} \in\left(\mathcal{N}_{-k} C_{k}\left(X_{0}\right)\right)^{G}$ de $c$ dans $X_{0}$. Si l'on montre alors que, dans ces conditions, il existe $\gamma_{0} \in \mathcal{N}_{-k+1} C_{k}\left(X_{0}\right)$ tel que $\bar{c}=\bar{c}_{\mid X_{0}^{G}}+(1+\sigma) \gamma_{0}$, on obtient par restriction

$$
c=\bar{c}_{\mid X}=c_{\mid X^{G}}+(1+\sigma) \gamma_{0 \mid X}
$$

avec $\gamma_{0 \mid X} \in \mathcal{N}_{-k+1} C_{k}(X)$.

(2) On suppose ensuite que $X$ est l'adhérence de Zariski $\bar{M}^{\mathcal{Z}}$ de $M$ et qu'en particulier $\operatorname{dim} X=k$. En effet, si l'on note $Z:=\bar{M}^{\mathcal{Z}}$, alors $Z$ est un fermé de $X$ globalement invariant sous l'action de $G$ (car $M$ l'est) et, comme par définition Supp $c \subset Z$, on a $c \in \mathcal{N}_{-k} C_{k}(Z)$.

(3) On peut aussi supposer que la variété $X$ est non-singulière, en considérant une résolution équivariante $\pi: \widetilde{X} \rightarrow X$ des singularités de $X$ ([7], 4] Appendix) et le tiré en arrière $\widetilde{c}:=\pi^{-1} c \in$ $\left(\mathcal{N}_{-k} C_{k}(\widetilde{X})\right)^{G}$ de $c$. Alors, si l'on montre que dans ces conditions, $\widetilde{c}=\widetilde{c}_{\mid \widetilde{X}^{G}}+(1+\sigma) \widetilde{\gamma}$ avec $\widetilde{\gamma} \in \mathcal{N}_{-k+1} C_{k}(\widetilde{X})$, on obtient, en poussant en avant,

$$
c=\pi_{*}(c)=c_{\mid X^{G}}+(1+\sigma) \pi_{*}(\widetilde{\gamma})
$$

(avec $\pi_{*}(\widetilde{\gamma}) \in \mathcal{N}_{-k+1} C_{k}(X)$ ), car $\pi$ est un isomorphisme en dehors d'une sous-variété de codimension au moins 1 et $c$ est de dimension $k=\operatorname{dim} X)$. 
Au total, la chaîne $c$ étant pure, on peut supposer que le support $M$ de $c$ est un sous-ensemble symétrique par arcs (dont toutes les composantes connexes sont) de dimension maximale d'une variété compacte non-singulière $X$, i.e. une union de composantes connexes de dimension maximale de $X([10])$.

(4) Enfin, l'involution $\sigma$ échange ou fixe globalement les cartes affines recouvrant $X$, ainsi que les composantes connexes de $X$, et donc les restrictions de $M$ à celles-ci, qui constituent également des chaînes pures de $X$. On peut donc supposer sans perdre de généralité que $M$ est une composante connexe d'un sous-ensemble algébrique compact non-singulier d'un espace affine, globalement stable sous l'action (non triviale) de $\sigma$, qui peut être considérée comme une sous-variété Nash compacte connexe d'un espace affine.

On utilise alors le théorème 4.1 pour écrire

$$
[M]=(1+\sigma)[A]
$$

dans $C_{k}(X)$ (l'ensemble des points invariants $M^{G}$ est de codimension au moins 1 comme l'action de $G$ n'est pas triviale, donc $\left[M^{G}\right]=0$ dans $\left.C_{k}(X)\right)$, avec $[A] \in C_{k}(X)$ vérifiant $\partial[A]=[\partial(A)] \in$ $\mathcal{N}_{-k+1} C_{k-1}(X)$, i.e. $[A] \in \mathcal{N}_{-k+1} C_{k}(X)$ (on utilise l'une des équivalences de la preuve de [14] Corollary 3.12: $X$ est compacte non-singulière de dimension $k$ ).

On va alors utiliser le découpage des chaînes pures pour découper les chaînes de degré plus élevé dans la filtration. Précisément, pour couper en deux une chaîne invariante sous l'action de $G$, de façon à contrôler la "régularité" des parties que l'on obtient, il suffit de découper l'adhérence de Zariski de son support en utilisant la propriété précédente :

Proposition 5.2. Soit $c \in\left(\mathcal{N}_{\alpha} C_{k}(X)\right)^{G}$ une k-chaîne quelconque de $X$, invariante sous l'action de l'involution $\sigma$. Alors on peut écrire

$$
c=c_{\mid X^{G}}+(1+\sigma) c^{\prime}
$$

avec $c_{\mid X^{G}} \in \mathcal{N}_{\alpha} C_{k}\left(X^{G}\right)$ et $c^{\prime} \in \mathcal{N}_{\alpha+1} C_{k}(X)$

Démonstration. On note $c^{\mathcal{Z}}$ la chaîne de $C_{k}(X)$ représentée par l'adhérence de Zariski du support noté $A$ de la chaîne $c$. On a alors $c^{\mathcal{Z}} \in\left(\mathcal{N}_{-k} C_{k}(X)\right)^{G}$ et, d'après la propriété précédente, il existe $\gamma \in \mathcal{N}_{-k+1} C_{k}(X)$ tel que

$$
c^{\mathcal{Z}}=\left(c^{\mathcal{Z}}\right)_{\mid X^{G}}+(1+\sigma) \gamma
$$

Revenons à la définition de la filtration Nash-constructible (14] section 3) : soit donc une fonction $\psi: X \rightarrow 2 \mathbb{Z}$ génériquement Nash-constructible en dimension $k$ telle que $\gamma=[S]$ avec

$$
S=\left\{x \in X \mid \psi(x) \notin 2^{2} \mathbb{Z}\right\},
$$

et soit une fonction $\varphi: X \rightarrow 2^{k+\alpha} \mathbb{Z}$ génériquement Nash-constructible en dimension $k$ telle que $c=[B]$ avec

$$
B=\left\{x \in X \mid \varphi(x) \notin 2^{k+\alpha+1} \mathbb{Z}\right\} .
$$


On considère alors le produit $\varphi \times \psi: X \rightarrow 2^{k+\alpha+1} \mathbb{Z}$ qui est une fonction génériquement Nash constructible en dimension $k$ (comme produit de telles fonctions). On a

$$
\begin{aligned}
B \cap S & =\left\{x \in X \mid \varphi(x) \notin 2^{k+\alpha+1} \mathbb{Z} \text { et } \psi(x) \notin 2^{2} \mathbb{Z}\right\} \\
& =\left\{x \in X \mid \varphi \times \psi(x) \notin 2^{k+\alpha+2} \mathbb{Z}\right\} .
\end{aligned}
$$

On note $c^{\prime}$ la chaîne représentée par $B \cap S$, qui appartient donc à $\mathcal{N}_{\alpha+1} C_{k}(X)$, et on a

$$
\begin{aligned}
(1+\sigma) c^{\prime} & =[B \cap S]+[\sigma(B) \cap \sigma(S)] \\
& =[B \cap S]+[B \cap \sigma(S)](\text { lemme 2.6) } \\
& =[c l(B \cap(S \div \sigma(S)))] \\
& =[B \cap c l(S \div \sigma(S))] \text { (lemme 2.7) }
\end{aligned}
$$

or $[B]=[A]$ et

$$
[c l(S \div \sigma(S))]=(1+\sigma)[S]=c^{\mathcal{Z}}+\left(c^{\mathcal{Z}}\right)_{\mid X^{G}}=\left[c l\left(\bar{A}^{\mathcal{Z}} \backslash \bar{A}^{\mathcal{Z}} \cap X^{G}\right)\right],
$$

donc

$$
\begin{aligned}
(1+\sigma) c^{\prime} & =\left[A \cap c l\left(\bar{A}^{\mathcal{Z}} \backslash \bar{A}^{\mathcal{Z}} \cap X^{G}\right)\right] \text { (lemme 2.6) } \\
& =\left[\operatorname{cl}\left(A \cap\left(\bar{A}^{\mathcal{Z}} \backslash \bar{A}^{\mathcal{Z}} \cap X^{G}\right)\right)\right] \text { (lemme 2.7) } \\
& =\left[\operatorname{cl}\left(A \backslash A \cap X^{G}\right)\right] \\
& =c+c_{\mid X^{G}} .
\end{aligned}
$$

On résume cette propriété de découpage des chaînes invariantes par une suite exacte courte qui rappelle la suite exacte courte de Smith, que l'on adapte ici aux contraintes de la filtration Nash-constructible. On définit pour cela une nouvelle filtration avant de donner la suite exacte courte que l'on nommera Smith Nash-constructible :

Définition 5.3. Pour tout $k$ et tout $\alpha$, on note

$$
T_{k}^{\alpha+1}(X):=\left\{c \in \mathcal{N}_{\alpha+1} C_{k}(X) \mid(1+\sigma) c \in \mathcal{N}_{\alpha} C_{k}(X)\right\} .
$$

Remarque 5.4. Pour tout $\alpha, T_{*}^{\alpha+1}(X)$, muni de la différentielle induite par celle de $C_{*}(X)$, est un complexe de chaînes. On obtient ainsi une nouvelle filtration

$$
0=T_{k}^{-k-1}(X) \subset T_{k}^{-k}(X) \subset \cdots \subset T_{k}^{1}(X)=C_{k}(X)
$$

de $C_{*}(X)$.

Théorème 5.5. Pour tout $\alpha$, la suite de complexes

$$
0 \rightarrow \mathcal{N}_{\alpha} C_{*}\left(X^{G}\right) \oplus(1+\sigma) T_{*}^{\alpha+1}(X) \rightarrow \mathcal{N}_{\alpha} C_{*}(X) \rightarrow(1+\sigma) \mathcal{N}_{\alpha} C_{*}(X) \rightarrow 0,
$$

est exacte.

On l'appelle suite exacte courte de Smith Nash-constructible de degré $\alpha$. 
On peut interpréter cette suite exacte dans le cas où $X$ est une variété compacte munie d'une action libre de $G$. En effet, dans ces conditions, on a un isomorphisme filtré entre les chaînes invariantes et les chaînes du quotient :

Proposition 5.6. Supposons que la G-variété algébrique réelle $X$ soit compacte, et que l'action de $G$ sur $X$ soit libre. Alors le quotient $X / G$ (qui désigne par abus de notation le quotient de l'ensemble des points réels de $X$ par l'action de $G$ restreinte) est un ensemble symétrique par arcs ([5] Proposition 3.15) et, pour tous $k, \alpha \in \mathbb{Z}$, on a un isomorphisme

$$
\left(\mathcal{N}_{\alpha} C_{k}(X)\right)^{G} \cong(1+\sigma) T_{k}^{\alpha+1}(X) \cong \mathcal{N}_{\alpha} C_{k}(X / G) .
$$

Démonstration. Fixons $k$ et $\alpha$. Notons tout d'abord que la suite exacte de Smith Nash-constructible nous donne le premier isomorphisme, étant donné que l'action de $G$ sur $X$ est libre.

Pour montrer le second isomorphisme, on considère d'une part le morphisme

$$
1+\sigma: T_{k}^{\alpha+1}(X) \rightarrow T_{k}^{\alpha+1}(X)
$$

dont le noyau est $\left(T_{k}^{\alpha+1}(X)\right)^{G}$ et l'image est $(1+\sigma) T_{k}^{\alpha+1}(X)$, et d'autre part le morphisme

$$
T_{k}^{\alpha+1}(X) \stackrel{\pi_{*}}{\rightarrow} \mathcal{N}_{\alpha+1} C_{k}(X / G),
$$

obtenu par restriction à $T_{k}^{\alpha+1}(X)$ du morphisme $\mathcal{N}_{\alpha+1} C_{k}(X) \rightarrow \mathcal{N}_{\alpha+1} C_{k}(X / G)$, induit par l'application quotient $X \rightarrow X / G$ (qui est une application propre continue de graphe $\mathcal{A S}$ ).

On montre par la suite que le noyau de $\pi_{*}$ est $\left(T_{k}^{\alpha+1}(X)\right)^{G}$ et que son image est $\mathcal{N}_{\alpha} C_{k}(X / G)$. Ainsi, les morphismes $1+\sigma$ et $\pi_{*}$ possédant les mêmes noyaux (et les mêmes espaces de départ), on obtient un isomorphisme entre leurs images, soit

$$
(1+\sigma) T_{k}^{\alpha+1}(X) \cong \mathcal{N}_{\alpha} C_{k}(X / G) .
$$

Montrons donc dans un premier temps que le noyau de $\pi_{*}$ consiste en les chaînes invariantes de $T_{k}^{\alpha+1}(X)$ (qui sont les exactement les chaînes invariantes de $\mathcal{N}_{\alpha+1} C_{k}(X)$ ).

Soit $c \in T_{\alpha+1}$ tel que $\pi_{*} c=0$. Si $c=\operatorname{Supp} \varphi_{c, \alpha} \bmod 2^{k+\alpha+2}$ avec $\varphi_{c, \alpha}: X \rightarrow 2^{k+\alpha+1} \mathbb{Z}$ génériquement Nash-constructible en dimension $k$, alors

$$
\pi_{*} c=\operatorname{Supp} \pi_{*}\left(\varphi_{c, \alpha}\right) \bmod 2^{k+\alpha+2}
$$

où $\pi_{*}\left(\varphi_{c, \alpha}\right): X / G \rightarrow 2^{k+\alpha+1} \mathbb{Z}$ est une fonction génériquement Nash-constructible en dimension $k$ sur $X / G([14$ Corollary 3.5).

Or, pour toute fonction Nash-constructible $f$ sur $X$, comme l'action de $\sigma$ sur $X$ est libre, $\pi_{*}(f)(\bar{x})=f(x)+f(\sigma(x))$ pour tout point $\bar{x} \in X / G$. Ainsi,

$$
\pi_{*} c=\left\{\bar{x} \in X / G \mid \varphi(x)+\varphi(\sigma(x)) \notin 2^{k+\alpha+2} \mathbb{Z}\right\},
$$

$\operatorname{avec} \varphi:=\varphi_{c, \alpha}$. 
Par hypothèse cette chaîne est nulle, i.e. pour tout $\bar{x}$ en dehors d'un sous-ensemble semialgébrique de $X / G$ de dimension $<k$ (i.e. pour tout $x$ en dehors d'un sous-ensemble semialgébrique de $X$ de dimension $<k), \varphi(x)+\varphi(\sigma(x)) \in 2^{k+\alpha+2} \mathbb{Z}$. En particulier, en dehors d'un sous-ensemble semi-algébrique de codimension au moins 1 , tout point du représentant $\left\{x \in X \mid \varphi(x) \notin 2^{k+\alpha+2} \mathbb{Z}\right\}$ de la chaîne $c$ vérifie à la fois $\varphi(x) \notin 2^{k+\alpha+2} \mathbb{Z}$ (par définition) et $\varphi(x)+\varphi(\sigma(x)) \in 2^{k+\alpha+2} \mathbb{Z}$, et donc également $\varphi(\sigma(x)) \notin 2^{k+\alpha+2} \mathbb{Z}$. Or $\varphi \circ \sigma=\sigma^{*}(\varphi)$ représente la chaîne $\sigma c$, et la chaîne $c$ est donc invariante sous l'action de $\sigma$.

On a donc ker $\pi_{*} \subset\left(T_{k}^{\alpha+1}(X)\right)^{G}$. Réciproquement, si $c \in\left(T_{k}^{\alpha+1}(X)\right)^{G}$, alors, en reprenant les notations ci-dessus, $\varphi(x) \notin 2^{k+\alpha+2} \mathbb{Z}$ et $\varphi(\sigma(x)) \notin 2^{k+\alpha+2} \mathbb{Z}$ pour tout $x$ dans le support de $c$, génériquement en dimension $k$, et donc $\varphi(x)+\varphi(\sigma(x)) \in 2^{k+\alpha+2} \mathbb{Z}$. Ainsi, $\pi_{*} c=0$

Montrons maintenant que l'image de $\pi_{*}$ est constituée des chaînes de degré $\alpha$ du quotient $X / G$.

Soit $c \in \mathcal{N}_{\alpha} C_{k}(X / G)$ et soit $\varphi: X / G \rightarrow 2^{k+\alpha} \mathbb{Z}$ génériquement Nash-constructible en dimension $k$ telle que

$$
c=\left\{\bar{x} \in X / G \mid \varphi(\bar{x}) \notin 2^{k+\alpha+1} \mathbb{Z}\right\} .
$$

On considère la chaîne, que l'on note $\pi^{*} c$, représentée par l'ensemble semi-algébrique de dimension $k$

$$
\left\{x \in X \mid \pi^{*}(\varphi)(x) \notin 2^{k+\alpha+1} \mathbb{Z}\right\}=\pi^{-1}\left(\left\{\bar{x} \in X / G \mid \varphi(\bar{x}) \notin 2^{k+\alpha+1} \mathbb{Z}\right\}\right)
$$

où le tiré en arrière $\pi^{*}(\varphi): X \rightarrow 2^{k+\alpha} \mathbb{Z}$ ([14] Corollary 3.5) est une fonction génériquement Nash-constructible en dimension $k$ telle que, pour $x \in X$ en dehors d'un sous-ensemble semialgébrique de dimension $<k, \pi^{*}(\varphi)(x)=\varphi(\pi(x))=\varphi(\bar{x})$.

La chaîne $\pi^{*} c$ appartient donc à $\mathcal{N}_{\alpha} C_{k}(X)$ et est de plus invariante sous l'action de $G$. En suivant le raisonnement qui nous a menés à l'exactitude de la suite de Smith Nash-constructible, on sait que l'on peut écrire $\pi^{*} c=(1+\sigma) \gamma$ avec

$$
\gamma=\left\{x \in X \mid \psi(x) \notin 2^{2} \mathbb{Z} \text { et } \pi^{*}(\varphi)(x) \notin 2^{k+\alpha+1} \mathbb{Z}\right\}
$$

où $\psi: X \rightarrow 2 \mathbb{Z}$ est une fonction génériquement Nash-constructible en dimension $k$, dont le support modulo $2^{2}$ représente l'une des deux parties, images l'une de l'autre par $\sigma$, que l'on a obtenues par découpage de la chaîne représentée par l'adhérence de Zariski du support de $\pi^{*} c$.

On applique alors $\pi_{*}$ à $\gamma$ :

$$
\begin{aligned}
\pi_{*} \gamma & =\left\{\bar{x} \in X / G \mid \pi_{*}\left(\psi \times \pi^{*}(\varphi)\right)(\bar{x}) \notin 2^{k+\alpha+2} \mathbb{Z}\right\} \\
& =\left\{\bar{x} \in X / G \mid \varphi(\bar{x})(\psi(x)+\psi(\sigma(x))) \notin 2^{k+\alpha+2} \mathbb{Z}\right\} .
\end{aligned}
$$

Si $\bar{x} \in X / G$ vérifie $\varphi(\bar{x})(\psi(x)+\psi(\sigma(x))) \notin 2^{k+\alpha+2} \mathbb{Z}$, alors $\varphi(\bar{x}) \notin 2^{k+\alpha+1} \mathbb{Z}$. Réciproquement, si $\bar{x} \in X / G$ vérifie $\varphi(\bar{x}) \notin 2^{k+\alpha+1} \mathbb{Z}$, alors $x$ appartient au support de $\pi^{*} c$ (vrai au moins génériquement en dimension $k$ ) et donc soit au support de $\gamma$ soit au support de $\sigma \gamma$, mais pas aux deux, et ainsi $\psi(x)+\psi(\sigma(x)) \notin 2^{2} \mathbb{Z}$ et donc $\varphi(\bar{x})(\psi(x)+\psi(\sigma(x))) \notin 2^{k+\alpha+2} \mathbb{Z}$ (génériquement).

On obtient alors

$$
\pi_{*} \gamma=\left\{\bar{x} \in X / G \mid \varphi(\bar{x}) \notin 2^{k+\alpha+1}\right\}=c
$$


et $c$ appartient donc à l'image de $T_{k}^{\alpha+1}(X)$ par $\pi_{*}$.

Réciproquement, soit $c \in T_{k}^{\alpha+1}(X)$. On a $c=\operatorname{Supp} \psi \bmod 2^{k+\alpha+2}$ avec $\psi: X \rightarrow 2^{k+\alpha+1} \mathbb{Z}$ génériquement Nash-constructible en dimension $k$, et $(1+\sigma) c=\operatorname{Supp} \varphi \bmod 2^{k+\alpha+1}$ avec $\varphi$ génériquement Nash-constructible en dimension $k$. Comme $(1+\sigma) c=\operatorname{Supp} \frac{\psi+\psi \circ \sigma}{2} \bmod 2^{k+\alpha+1}$, on a alors

$$
\varphi \equiv \frac{\psi+\psi \circ \sigma}{2} \bmod 2^{k+\alpha+1}
$$

sur $X$.

On considère maintenant

$$
\pi_{*} c=\left\{\bar{x} \in X / G \mid \pi_{*}(\psi)(\bar{x}) \notin 2^{k+\alpha+2}\right\}=\left\{\bar{x} \in X / G \mid \psi(x)+\psi(\sigma(x)) \notin 2^{k+\alpha+2}\right\} .
$$

On montre que l'on peut trouver une fonction génériquement Nash-constructible sur $X / G$, divisible par $2^{k+\alpha}$, qui représente $\pi_{*} c$. Pour cela, on applique le critère de l'éventail [14 Theorem 4.10 à chacune des composantes irréductibles de chaque carte affine de l'adhérence de Zariski $Y$ de $X / G$ et à la fonction

$$
f: y \mapsto\left\{\begin{array}{l}
\frac{\psi(x)+\psi(\sigma(x))}{2} \text { si } y=\bar{x} \in X / G, \\
0 \text { sinon, }
\end{array}\right.
$$

définie et constructible sur $Y$.

Soit donc $F$ un éventail centré en un point $y_{0}$ de $Y$, de cardinal $|F| \leq 2^{k+\alpha+1}$. Comme $X$ est compact et l'action de $G$ sur $X$ étant libre, si le point $y_{0}=\overline{x_{0}}$ est dans $X / G$, on peut relever $F$ en un éventail double $F^{\prime} \sqcup \sigma\left(F^{\prime}\right)$ sur $X\left(F^{\prime}\right.$ étant centré en $x_{0}$ et $\sigma\left(F^{\prime}\right)$ en $\sigma\left(x_{0}\right)$ ), et sinon l'évaluation de $f$ en tout point de l'éventail sera nulle. Concentrons-nous donc sur le premier cas. On a alors

$$
\sum_{s \in F} f(s)=\sum_{s^{\prime} \in F^{\prime}} \frac{\psi\left(s^{\prime}\right)+\psi\left(\sigma\left(s^{\prime}\right)\right)}{2}
$$

et cette somme, modulo $|F|=\left|F^{\prime}\right| \leq 2^{k+\alpha+1}$, est égale à $\sum_{\sigma^{\prime} \in F^{\prime}} \varphi\left(\sigma^{\prime}\right)$ qui est elle-même égale à 0 modulo $\left|F^{\prime}\right| \leq 2^{k+\alpha+1}$, car $\varphi$ est génériquement Nash-constructible sur $X$ ([14] Theorem 4.9 d'après [2]).

Ainsi (14] Theorem 4.10), il existe une fonction $f_{0}: Y \rightarrow \mathbb{Z}$, génériquement Nash-constructible, telle que, pour tout $y \in Y, f(y)-f_{0}(y) \equiv 0 \bmod 2^{k+\alpha+1}$. En particulier, $f_{0}$ est divisible par $2^{k+\alpha}$ et

$$
\pi_{*} c=\operatorname{Supp} f_{0} \bmod 2^{k+\alpha+1} .
$$

On peut de plus supposer $f_{0}$ génériquement constructible en dimension $k$, en remplaçant, dans le raisonnement précédent, $X$ par l'adhérence de Zariski du support de $c$. On a donc $\pi_{*} c \in \mathcal{N}_{\alpha} C_{k}(X / G)$.

Remarque 5.7. - Les isomorphismes $\left(\mathcal{N}_{\alpha} C_{k}(X)\right)^{G} \cong \mathcal{N}_{\alpha} C_{k}(X / G)$ induisent des isomorphismes de complexes

$$
\left(\mathcal{N}_{\alpha} C_{*}(X)\right)^{G} \cong \mathcal{N}_{\alpha} C_{*}(X / G),
$$

induits par l'application quotient $\pi: X \rightarrow X / G$ par fonctorialité de $\mathcal{N}_{\alpha} C_{*}$. 
- Pour une $G$-variété algébrique réelle $X$ quelconque munie d'une action de $G$ quelconque, en raisonnant de façon similaire, on obtient également les isomorphismes (de complexes)

$$
(1+\sigma) \mathcal{N}_{\alpha} C_{*}(X) \cong i m\left(\mathcal{N}_{\alpha} C_{*}(X) \rightarrow C_{*}\left(X \backslash X^{G} / G\right)\right)
$$

(où $X \backslash X^{G} / G$ désigne l'ensemble des points réels de $X \backslash X^{G}$ quotienté par l'action restreinte de $G)$.

\section{Références}

[1] J. Bochnak, M. Coste, M.-F. Roy, Real Algebraic Geometry, Springer-Verlag, Berlin, 1998.

[2] I. Bonnard, Nash constructible functions, Manuscripta Math. 112 (2003), 55-75.

[3] P. Deligne, Poids dans la cohomologie des variétés algébriques, Proc. Int. Cong. Math. Vancouver (1974), 79-85.

[4] J. Denef, F. Loeser, Motivic Igusa zeta functions, J. Algebraic Geom. 7 (1998), no. 3, 505-537.

[5] G. Fichou, Equivariant virtual Betti numbers, Ann. de l'Inst. Fourier, 58, no. 1 (2008), $1-27$.

[6] F. Guillén, V. Navarro Aznar, Un critère d'extension des foncteurs définis sur les schémas lisses, IHES Publ. Math. 95 (2002), 1-83.

[7] H. Hironaka, Resolution of singularities of an algebraic variety over a field of characteristic zero, Ann. Math. 79 (1964), no. 2, 109-326.

[8] D. Husemöller, Fiber Bundles, Third Ed., Springer, New-York, 1994.

[9] K. Kurdyka, Ensembles semi-algébriques symétriques par arcs, Math. Ann. 281 (1988), 445-462.

[10] K. Kurdyka, A. Parusiński, Arc-symmetric sets and arc-analytic mappings, Panoramas et Synthèses 24, Soc. Math. France (2007), 33-67.

[11] J.M. Lee, Introduction to smooth manifolds, Springer-Verlag, New-York, 2003.

[12] C. McCrory, A. Parusiński, Algebraically constructible functions, Ann. Sci. cole Norm. Sup. 30 (1997), 527-552.

[13] C. McCrory, A. Parusiński, Virtual Betti numbers of real algebraic varieties, C. R. Math. Acad. Sci. Paris 336 (2003), no. 9, 763-768.

[14] C. McCrory, A. Parusiński, The weight filtration for real algebraic varieties, Topology of stratified spaces, 121-160, Math. Sci. Res. Inst. Publ. 58, Cambridge Univ. Press, Cambridge, 2011.

[15] C. Procesi, G. Schwarz, Inequalities defining orbit spaces, Invent. Math. 81 (1985), 539-554.

[16] M. Shiota, Nash manifolds, Lectures Notes in Math., 1269, Springer-Verlag, 1987.

[17] B. Totaro, Topology of singular algebraic varieties, Proc. Int. Cong. Math. Beijing (2002), $533-541$. 